\title{
Artificial anisotropic dielectric material for antenna polarization rotation
}

\author{
M. Berg ${ }^{1}$, T. Tuovinen ${ }^{1}$, and E. Salonen ${ }^{1}$ \\ ${ }^{1}$ University of Oulu, Finland
}

\begin{abstract}
In this paper, an uniaxial anisotropic material block composed of dielectric sheets is examined for the rotation of the tilt angle of the linear polarization. The main objective is to diminish the coupling between two antenna elements. A theoretical model for polarization rotation is briefly introduced. The anisotropic material parameters are derived using electromagnetic mixing formulas. The theoretically predicted operation is verified by $3 \mathrm{D}$ EM-simulations using two parallel dipole antennas at the frequency of $1.5 \mathrm{GHz}$. The simulated transmission coefficient $\left(S_{21}\right)$ values between dipoles indicate $>30 \mathrm{~dB}$ increase in isolation in comparison with a homogeneous isotropic material sample and in free space. Theoretical and simulation results are promising regardless of the anisotropic material in the near field of the antenna.
\end{abstract}

\section{INTRODUCTION}

Artificial anisotropic materials are metamaterials with tailored properties e.g. for electromagnetic wave manipulation. Anisotropic materials enable direction-dependent behaviour for electromagnetic wave. An interesting application field is the polarization manipulation of the wave using anisotropic materials [1]-[2]. Ordinary methods include resonance-based approach where either anisotropic metal plates [1] or dielectric sheet with conductive material inclusions [2] are used for the polarization modification. These methods enable anisotropic behaviour for either reflected or transmitted wave.

There has not been such high interest on the dielectric anisotropic materials application in electromagnetic wave manipulation. A parametrical study on the effect of anisotropic antenna substrate and superstrate on the radiation of Hertzian dipole has been conducted [3], which indicates the increased number of design parameters for the antenna. Macroscopic scale anisotropy of dielectrics can be used to manipulate the radiation pattern of an on-ground antenna [4] via changing the polarization of the ground reflection. Generally, the behaviour of a homogenous bulk model of anisotropic material has been shown to correlate to that of the anisotropic substrate formed by metal inclusions [5].

The dielectric sheet -based approach has been considered earlier for a GPS L1 band monopole antenna in order to create an anisotropic antenna substrate, which changes the linear polarization to circular [6]. Also, an aperture coupled slot has been loaded by an anisotropic superstrate to modify polarization from linear to circular at $28 \mathrm{GHz}$ [7].

In this paper, an artificial dielectric metamaterial realization is introduced to manipulate the polarization properties of an electromagnetic wave. The macroscopic scale anisotropy is based on the layered structure of dielectric sheets. The anisotropic material is theoretically modelled and its operation for polarization rotation is verified by full-wave EM simulations. Furthermore, the material block dimensions are optimized by simulations in order to minimize coupling between two parallel dipole antennas.

\section{EFFECT OF ANISOTROPIC DIELECTRIC MATERIAL ON WAVE POLARIZATION}

The electric field component $\vec{E}$ of the electromagnetic wave propagating to $z$-direction can be divided into two orthogonal basis vectors, which are aligned according to the principal $x$ - and $y$ coordinates (shown in Fig. 1). Due to the unequal permittivity dyads of the anisotropic material $\left(\epsilon_{x}\right.$ and $\epsilon_{y}$ ), the difference in wave velocity for $\vec{E}_{x}$ and $\vec{E}_{y}$ is observed, and hence change in relative phases. Thus, the sum vector $\overrightarrow{E_{t o t}}=\overrightarrow{E_{x}}+\overrightarrow{E_{y}}$, i.e. the polarization of the wave is a function of $\epsilon_{r}$ values and the propagated distance. By selecting the thickness of the anisotropic medium such that $180^{\circ}$ phase difference is achieved between the field components, the tilt angle of the linearly polarized wave is rotated by $90^{\circ}$. The thickness of the anisotropic material block for the phase difference $P_{\text {dif }}=180^{\circ}$ is calculated by 


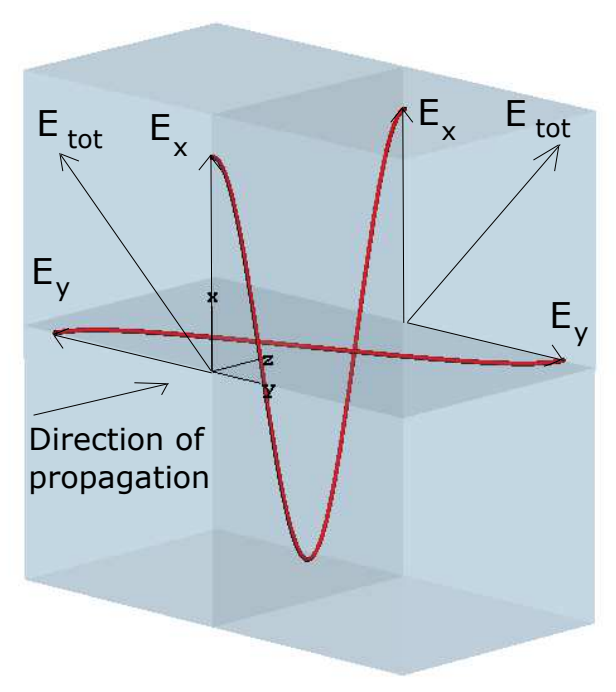

Figure 1: Polarization rotation due to propagation velocity difference between $\vec{E}_{x}$ and $\vec{E}_{y}$ in anisotropic material, $\epsilon_{x}<\epsilon_{y}$.

$$
D=\frac{\lambda_{0}}{\sqrt{\epsilon_{e f f, y}}-\sqrt{\epsilon_{e f f, x}}} \frac{P_{d i f}}{2 \pi},
$$

where $\lambda_{0}$ equals the free space wavelength and $\epsilon_{e f f, x}<\epsilon_{e f f, y}[6]$. Parameters for dimensioning correct material behaviour are relative effective permittivities in the direction of $x$ and $y$.

\section{DIELECTRIC SHEET APPROACH BASED ON MIXING FORMULAS}

The stacked dielectric sheet medium has been found to behave as homogeneous medium in macroscopic scale, when the thickness of individual sheet is small in wavelengths, e.g. $t_{\text {sheet }}<\lambda / 10$ [8]. The theoretical background of the layered dielectric sheet based anisotropic material for the polarization rotation of the wave is presented in [6]. However, the main points are presented here for better understanding and dielectric sheet dimensioning.

The physical background to the dielectric anisotropy is due to the polarizability $\alpha$ of a particle in an external electric field $\overrightarrow{E_{\text {ext }}}$ which cause dipole moment [9]

$$
\vec{p}=\alpha \overrightarrow{E_{\text {ext }}} .
$$

Polarizability of the particle depends on its shape [10]. Ellipsoidal particle polarizability in different directions can be defined using depolarization factors $\left(N_{x}, N_{y}, N_{z}\right)$ and they satisfy $N_{x}+$ $N_{y}+N_{z}=1$ for any ellipsoid. The higher depolarization factor gives smaller polarizability for the particle. For the uniaxial anisotropic behaviour of the material, one of the depolarization factors must differ from the other two. Thus, for maximum anisotropy the depolarization factors $N_{x}=1, N_{y}=0$ and $N_{z}=0$ are selected. These correspond a special case of ellipsoid, which is a thin disc. [9]

Maxwell Garnett electromagnetic mixing formula [11] is used to calculate the permittivity tensors for the dielectric sheet -based anisotropic material. In its classical form, the mixing formula for effective relative permittivity in the $x$-direction is

$$
\epsilon_{e f f, x}=\epsilon_{e}+v \epsilon_{e} \frac{\epsilon_{i}-\epsilon_{e}}{\epsilon_{e}+(1-v) N_{x}\left(\epsilon_{i}-\epsilon_{e}\right)},
$$

where $\epsilon_{e}$ and $\epsilon_{i}$ are dielectric constants for the environment and inclusion materials, respectively, and $v$ is the volume fraction of the inclusion material. The $y$ - and $z$-directions are calculated accordingly. In practise, Eq. 3 is used to calculate effective permittivity $\epsilon_{e f f, x}$ and $\epsilon_{e f f, y}$ which determine the polarization tilt angle rotation, shown in Fig. 1 and formulated in Eq. 1.

It is obvious that maximizing the square root difference in Eq. 1 yield to maximum polarization rotation, i.e. maximum difference between wave velocities. The effective values of $\epsilon_{x}$ and $\epsilon_{y}$ as a function of volume factor $v$ of inclusion material $\left(\epsilon_{i}=9.6\right)$ are shown in Fig. 2 when the 

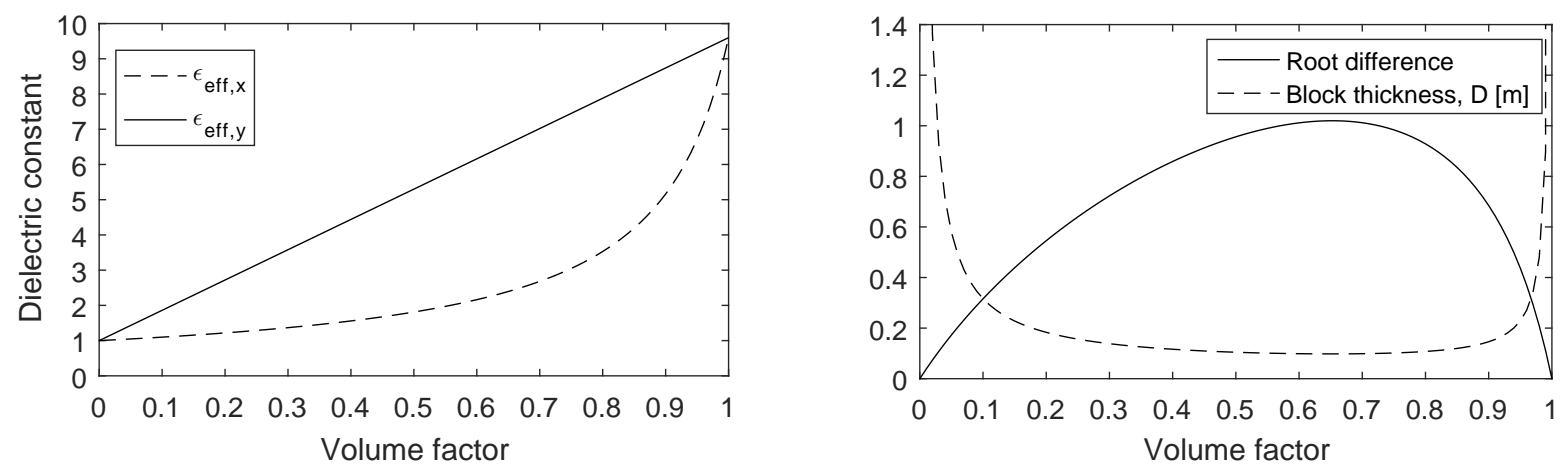

Figure 2: Theoretically calculated $\epsilon_{e f f, x}$ and $\epsilon_{e f f, y}$ (left), and the root difference and the anisotropic material block thickness (right) as a function of volume factor
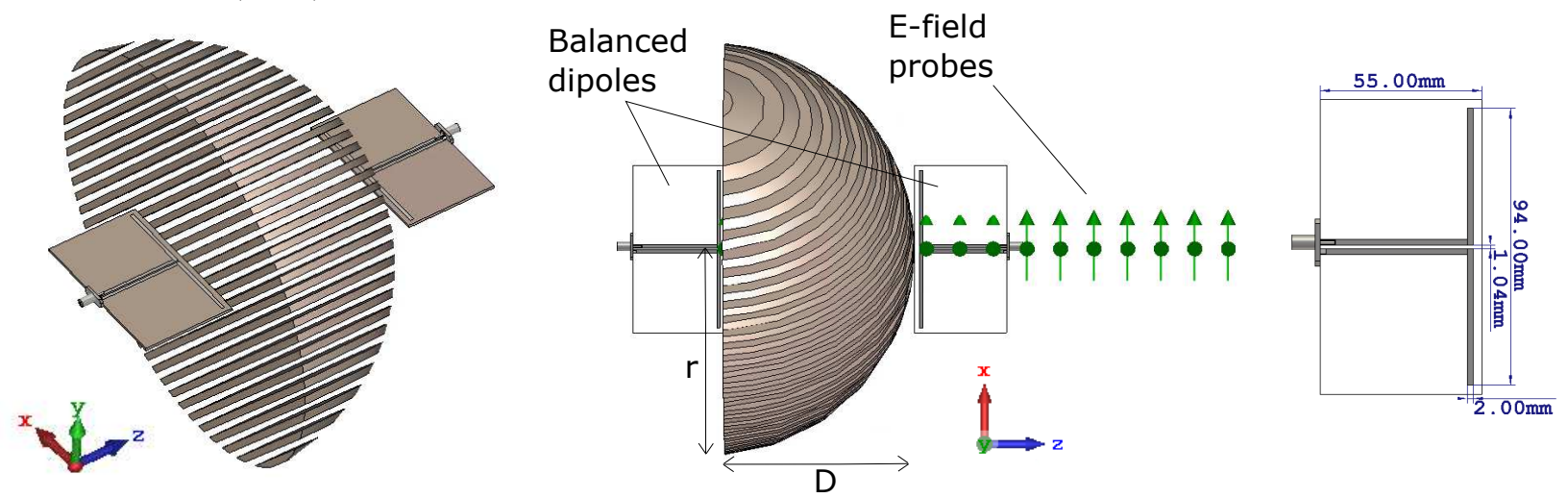

Figure 3: Oblique view of anisotropic material block and dipoles (left), top view with main dimensions (centre) and dimensions of the balanced dipole(right).

environmental material is air $\left(\epsilon_{e} \approx 1\right)$. The nonzero depolarization factor in Eq. 3 cause a nonlinear increase of $\epsilon_{e f f, x}$. Further, the square root difference and thickness of the anisotropic material block are calculated as a function of $v$ and shown in Fig. 2. The largest square root difference is achieved when $v=0.65$, which yields the block thickness of $0.098 \mathrm{~m}$ when phase difference of $180^{\circ}(\pi)$ is targeted. However, for EM-simulations, the volume factor $v=0.5$ was selected. Now, the theoretical thickness $D$ equals $0.104 \mathrm{~m}$ which is very close to the optimal, but enables more practical sheet thickness dimensioning. The sheet thickness of $4 \mathrm{~mm}$ was selected for both inclusion and environmental materials.

\section{3D EM SIMULATIONS FOR ANISOTROPIC MATERIAL}

An anisotropic material block was modelled using theoretical sheet thickness for environmental and inclusion materials. In order to achieve an equal distance for the spherically expanding wave inside anisotropic material, a segment of a sphere was selected as another surface of the material block as shown in Fig. 3.

The rotation of the polarization tilt angle of the wave was simulated by modelling two balanced dipoles and the anisotropic material block between dipoles in EM simulation software. The simulation model is shown in Fig. 3. Balanced dipole dimensions are for $\lambda / 2$ operation and dipole model includes PCB laminate material with the dielectric constant of 3.38, and the laminate thickness of $1.6 \mathrm{~mm}$. Dielectric sheets are aligned with $45^{\circ}$ angle relative to the main polarization plane of the dipole antenna. In simulations, two material block parameter values, the radius of the spherical block surface $(r)$ and the thickness of the block $(D)$, illustrated in Fig. 3, were varied to find the optimum behaviour. In simulations, the layered anisotropic material consisted of air $\left(\epsilon_{r 1}=1\right)$ and the dielectric sheets $\left(\epsilon_{r 2}=9.6, \tan \delta=0.008\right)$.

\section{ANISOTROPIC MATERIAL BLOCK PERFORMANCE AND ITS DIMENSIONING}

Transmission coefficient $\left(S_{21}\right)$ values between dipoles were used to indicate the polarization tilt angle rotation. The isolation was compared with an isotropic homogenous material block $\left(\epsilon_{r}=5.3\right)$ and 


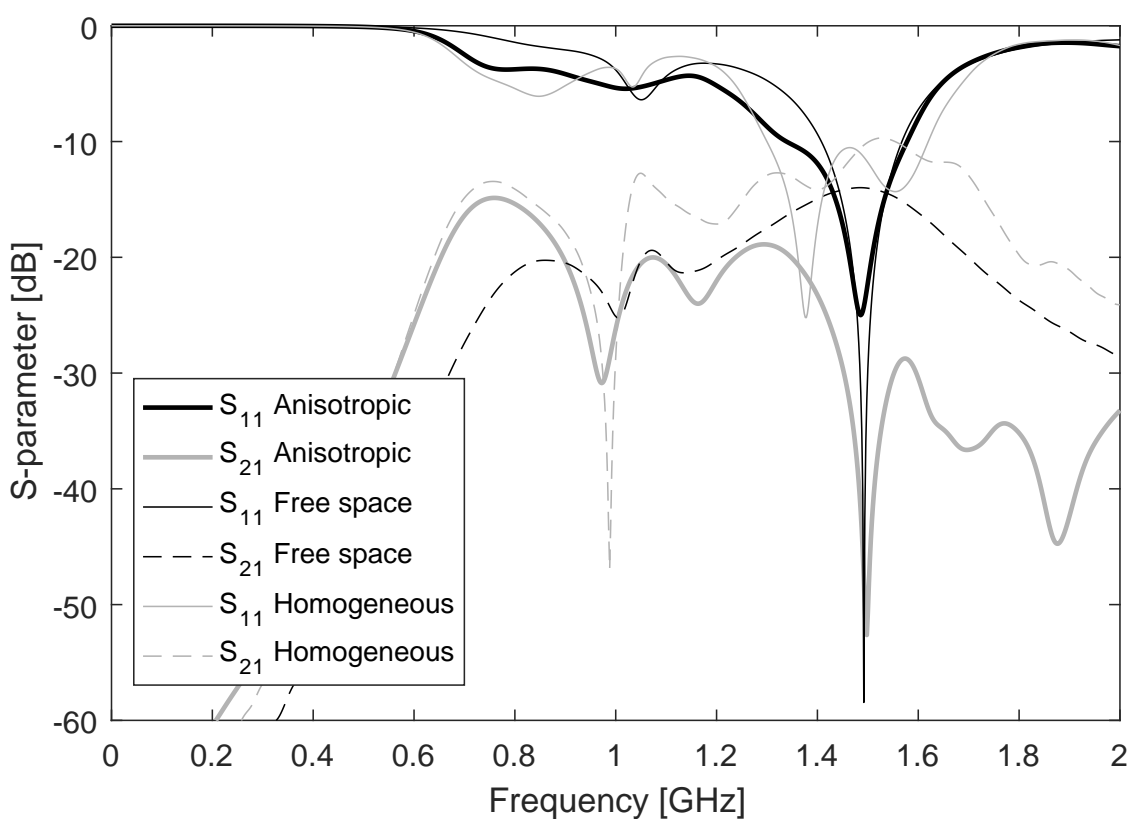

Figure 4: Simulated $S_{11}$ and $S_{21}$ parameters for anisotropic, homogeneous isotropic and free space propagation conditions.
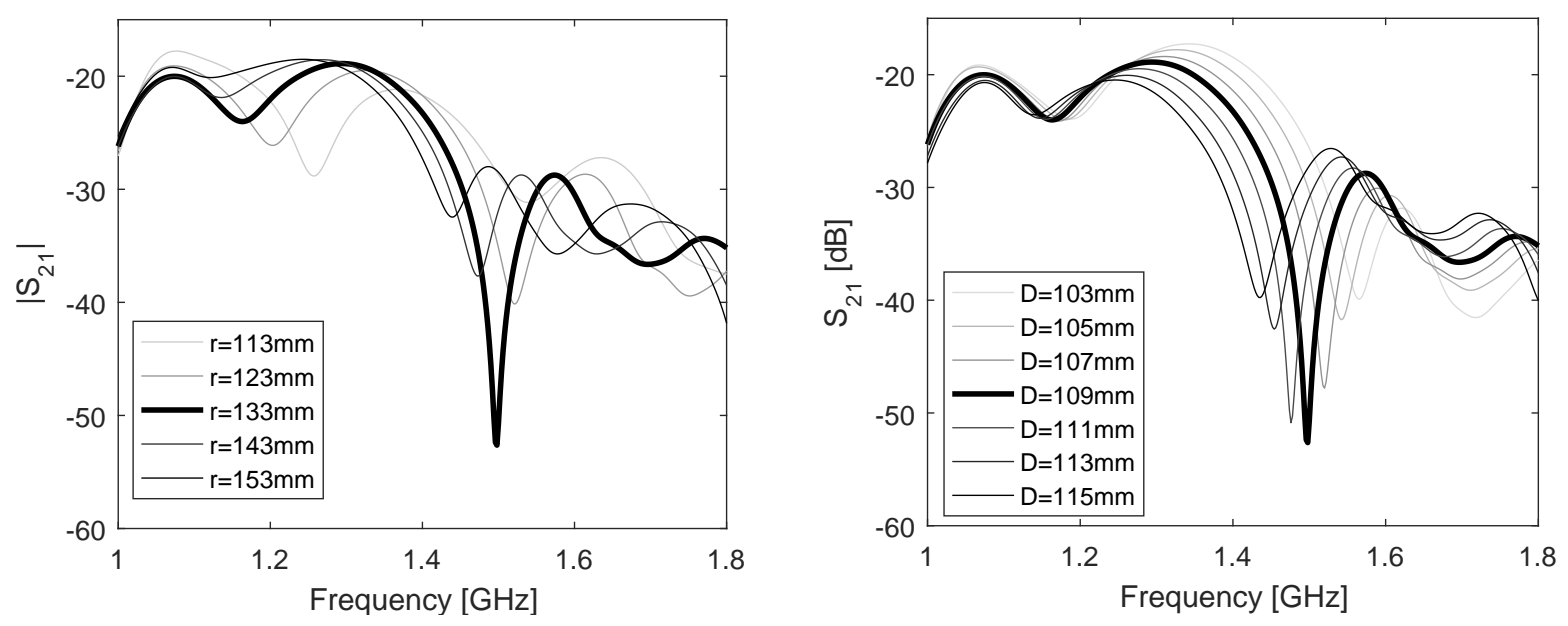

Figure 5: Effect of the surface radius $r$ on $S_{21}$ when $D=109 \mathrm{~mm}$ (left), and the effect of the material block thickness $D$ on $S_{21}$ when $r=133 \mathrm{~mm}$ (right).

with air (free space). Material blocks had identical outer shape and distance between antennas was kept equal.

The simulated transmission coefficient $\left(S_{21}\right)$ values between dipoles indicate $>30 \mathrm{~dB}$ increase in isolation at $1.5 \mathrm{GHz}$, due to anisotropy, in comparison with homogeneous isotropic material and in free space, as depicted in Fig. 4. The notably better isolation is achieved because of the rotation of the electromagnetic wave within the anisotropic medium. For the equal resonance frequency, as depicted by $S_{11}$ parameters in Fig. 4, the dipole length is increased from $94 \mathrm{~mm}$ to $101 \mathrm{~mm}$ for free space simulations, because of the excluded loading effect of the dielectric material block.

Influence of $r$ on $S_{21}$ is shown in Fig. 5. It is observed that high isolation between antennas is obtained when $r=133 \mathrm{~mm}$. With this radius, the centre of the spherical outer surface of the anisotropic block equals the phase centre of the transmit dipole. Approximately equal distance through the block creates the lowest $S_{21}$-parameter value.

Simulated $S_{21}$ parameters as a function of block thickness $D$ are shown in Fig. 5 . The highest isolation is achieved when $D=109 \mathrm{~mm}$, which is $5 \mathrm{~mm}$ more than theoretically predicted. Difference might be due to the material block location in the near field of the antenna, where E-field is a 

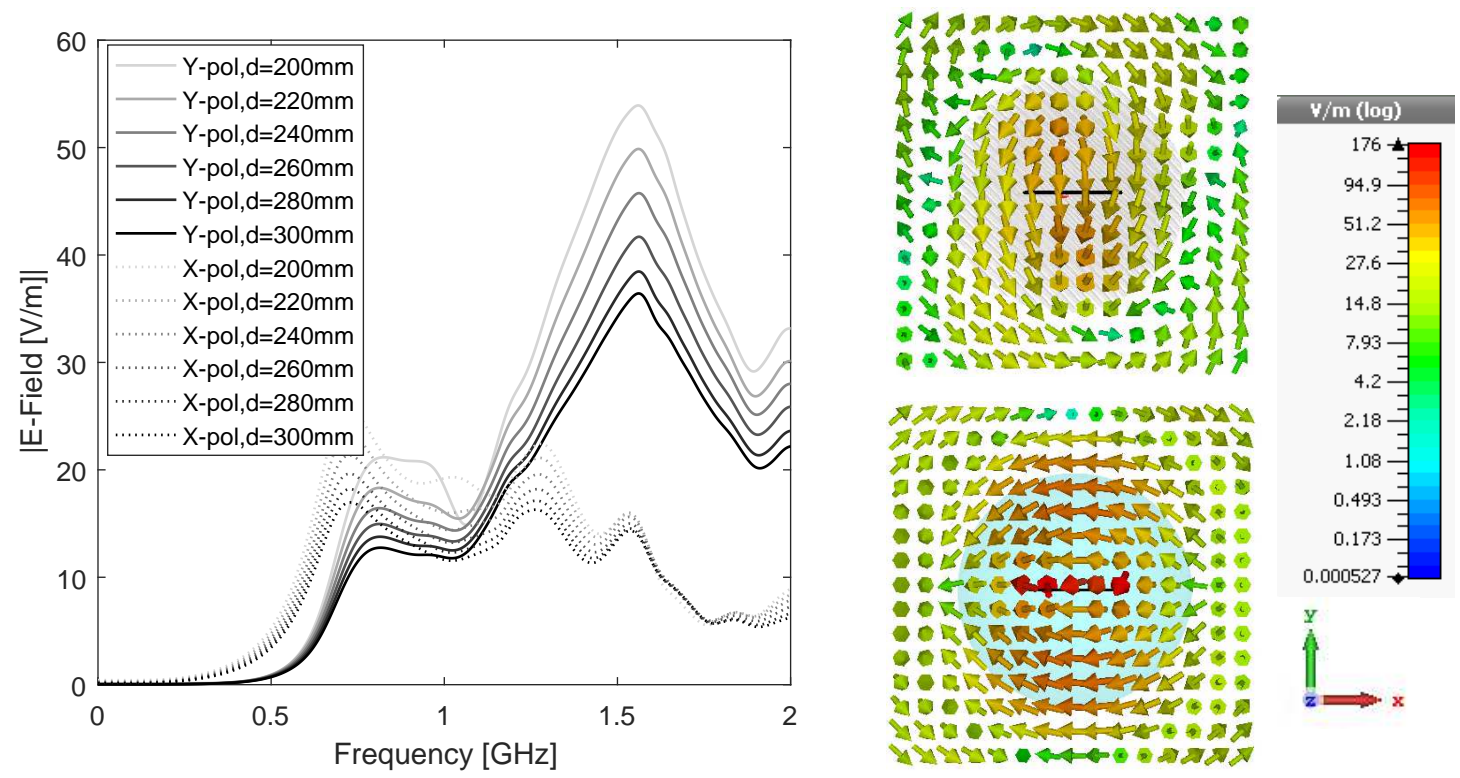

Figure 6: The $x$ - and $y$-directed E-field probe values for different distances from transmit antenna (left), and illustrated E-field vectors for anisotropic (right, top) and for homogeneous isotropic (right, bottom) material blocks.

function of distance from the antenna. Also, reflections from the boundary between the anisotropic material block and air may influence the received field polarization. The effect of reflections is minimized by optimizing the radius $r$ of the spherical surface of the material block.

The electric field probe (shown in Fig. 3) values for different distances from the transmitting dipole are shown in Fig. 6. Field values for $x$ - and $y$-directed probes show significant difference at $1.5 \mathrm{GHz}$, which indicate polarization rotation from $x z$-plane to $y x$-plane. Small decrease in $y$-polarized field value is observed as a function of distance. Electric field vector distributions in the $x y$-plane is illustrated in Fig. 6 for anisotropic, and for homogeneous isotropic material blocks at the receiving antenna (distance from transmit antenna $=109 \mathrm{~mm}$ ). Both transmit and receive antennas are positioned horizontally (in $x z$-plane), but orthogonally polarized wave can not be received in case of anisotropic material. Polarization rotation is due to correctly defined permittivity tensors based on the layered sheet method. For comparison, E-fields are parallel to the receiving antenna in case of isotropic material.

\section{DISCUSSION}

The presented uniaxial anisotropic material behaviour shows an interesting and low-loss way for the EM-wave polarization rotation. Although demonstrated here by using two parallel dipoles, a more interesting application area is the mutual coupling reduction in larger antenna arrays. The effect of anisotropy on the mutual coupling between dipoles has been investigated in [12]. However, the propagation delay based polarization vector rotation has not been examined. Low mutual coupling would enable a more precise beam forming and anisotropic dielectric could generate possibilities towards higher performance adaptive antenna arrays.

\section{CONCLUSION}

The theory and design of a simple uniaxial anisotropic material block for polarization rotation are described in this paper. The layered sheet method enables uniaxial anisotropic medium where dielectric constant tensors are controlled by sheet dielectric constants using electromagnetic mixing formulas. Theoretical examination is verified by EM simulations, where the anisotropic material block is located between parallel dipole antennas. A proper dimensioning of the anisotropic material block enables the polarization rotation between antennas and increase the antenna isolation over $30 \mathrm{~dB}$ in comparison with the free space or the isotropic material block. 


\section{REFERENCES}

1. Hao, J., Yuan, Y., Ran, L., Jiang, T., Kong, J. A., Chan, C. T. and Zhou, L."Manipulating Electromagnetic Wave Polarizations by Anisotropic Metamaterials," Phys. Rev. Lett., Vol. 99, 063908-1-063908-4, 2007.

2. Chen, M. L. N., Jiang, L. J., Sha, W. E. I., Choy, W. C. H. and Itoh, T., "Polarization Control by Using Anisotropic 3-D Chiral Structures," IEEE Trans. Antennas Propag., Vol. 64, No. 11, 4687-4694, 2016.

3. Eroglu, A. and Lee, J. K., "Far field radiation from an arbitrarily oriented Hertzian dipole in the presence of a layered anisotropic medium," IEEE Trans. Antennas Propag., Vol. 53, No. 12, 3963-3973, 2005.

4. Tuovinen, T., Berg, M. and Salonen, E. T. "Antenna Close to Tissue: Avoiding Radiation Pattern Minima With an Anisotropic Substrate," IEEE Antennas Wireless Propag. Lett., Vol. 13, 1680-1683, 2014.

5. Semichaevsky, A. and Akyurtlu, A., "Homogenization of metamaterial-loaded substrates and superstrates for antennas," Progress In Electromagnetics Research, Vol. 71, 129-147, 2007.

6. Tuovinen, T., Salonen, E. and Berg, M., "An artifically anisotropic antenna substrate for the generation of circular polarization," IEEE Trans. Antennas Propag, Vol. 64, No. 11, 4937-4942, 2016.

7. Tuovinen, T., Salonen, E. and Berg, M., "Artificially Anisotropic Substrate with ApertureCoupled Feeding for Generating Circularly-Polarized Antenna Solutions at mmWaves," Proceedings of LAPC, Loughborough, UK, November 2016, 1-4.

8. Collin, R. E., "A Simple Artificial Anisotropic Dielectric Medium," IRE Transactions on Microwave Theory and Techniques, vol. 6, no. 2, 206-209, 1958.

9. Sihvola, A. H., Electromagnetic mixing formulas and applications, IET, London, 1999.

10. Sihvola, A. H., "Dielectric Polarization and Particle Shape Effects," Journal of Nanomaterials, vol. 2007, 1-9, 2007.

11. Maxwell Garnett, J. C., "Colours in Metal Glasses and Metallic Films," Trans. of the Royal Society of London, vol. CCIII, 385-420, 1904.

12. Braaten, B. D., Rogers, D. A. and Nelson, R. M., "Multi-Conductor Spectral Domain Analysis of the Mutual Coupling Between Printed Dipoles Embedded in Stratified Uniaxial Anisotropic Dielectrics," IEEE Trans. Antennas Propag, Vol. 60, No. 4, 1886-1898, 2012. 